\title{
Optimization-based decision-making models for disaster recovery and reconstruction planning of transportation networks
}

\author{
Milad Zamanifar ${ }^{1} \cdot$ Timo Hartmann $^{1}$
}

Received: 19 December 2019 / Accepted: 16 July 2020 / Published online: 26 July 2020

(c) The Author(s) 2020

\begin{abstract}
The purpose of this study is to analyze optimization-based decision-making models for the problem of Disaster Recovery Planning of Transportation Networks (DRPTN). In the past three decades, seminal optimization problems have been structured and solved for the critical and sensitive problem of DRPTN. The extent of our knowledge on the practicality of the methods and performance of results is however limited. To evaluate the applicability of those context-sensitive models in real-world situations, there is a need to examine the conceptual and technical structure behind the existing body of work. To this end, this paper performs a systematic search targeting DRPTN publications. Thereafter, we review the identified literature based on the four phases of the optimization-based decision-making modeling process as problem definition, problem formulation, problem-solving, and model validation. Then, through content analysis and descriptive statistics, we investigate the methodology of studies within each of these phases. Eventually, we detect and discuss four research improvement areas as [1] developing conceptual or systematic decision support in the selection of decision attributes and problem structuring, [2] integrating recovery problems with traffic management models, [3] avoiding uncertainty due to the type of solving algorithms, and [4] reducing subjectivity in the validation process of disaster recovery models. Finally, we provide suggestions as well as possible directions for future research.
\end{abstract}

Keywords Disaster · Recovery · Reconstruction · Transportation network · Optimization

\section{Introduction}

Major natural, anthropogenic, or socio-natural hazards can create unnatural disasters in vulnerable exposed societies (Chmutina and Meding 2019). On the one hand, we might not be able to contain or restrain rapid-onset natural hazards such as earthquakes any soon. Nor did we manifest much achievement to stop triggering nature on the planet to avoid socio-natural hazards namely climate change-induced floods. On the other hand, mitigating

Milad Zamanifar

Milad.Zamanifar@gmail.com

1 Department of Civil Systems, Technische Universität Berlin, Gustav-Meyer-Allee 25, 13355 Berlin, Germany 
the exposure of critical infrastructures, as widely broadened networks on which our civilizations are built, comes at an extreme cost and thus renders the 'transferring risk' a disfavored solution. Consequently, in the context of infrastructures disaster risk reduction, alternatives are limited to two main concepts of 'reducing vulnerability' and 'increasing resiliency' of infrastructures. In urban areas, the transportation network is one of the most exposed physical infrastructures (Renne et al. 2020). However, pre-planning for post-event actions substantially increases the resiliency and capacity of the transportation network to recover from the devastating impacts of extreme natural phenomena (Naga and Fan 2007; Quarantelli 1999). The socioeconomic function of many individuals, enterprise, and critical services depends on the efficient operation of transportation networks (Cova and Cogner 2004). Therefore, successful planning for the disaster recovery process of transportation networks can be a central contributor to the resiliency of an urban area after hazard-induced disasters (Liao et al. 2018; Renne et al. 2020). This elaborates on the importance of conducting research in the field of Disaster Recovery Planning of Transportation Network (DRPTN). We define the decision-making model for DRPTN as a prescriptive decision model, constructed to respond to an extreme hazard that (1) exhibits structural damages on the transportation network's components. (2) Directly causes major operational disruptive impacts on the traffic functionally of the network or part of it and (3) can be alleviated only by physical construction interventions such as repair, and reconstruction operations.

Urban road networks and the traffic flow they operate are both vulnerable to natural hazards (Horner and Widener 2011). Meteorological hazards such as snow, avalanche, and storm mainly create closures, whereas flood as a hydrological hazard, earthquake as a tectonic hazard, and landslide as a cascade and/or geomorphological hazard cause destructions in network's components. While landslide is more expected in rural areas, urban transportation networks are mainly vulnerable due to fluvial floods, tsunamis, and seismic phenomena (Yin 2020; Koks et al. 2019). To impede the transformation of hazards to a disaster in the transportation system, owners and operators of infrastructures invest in optimized decisions for the recovery process to reduce the post-hazard downtime duration of road networks. Providing (ideally) low-cost high-impact solutions and accelerating recovery ratio as well as establishing rapid access to critical assets highlight the vital role of optimized planning for post-disaster recovery operations. The literature of DRPTN devotes to developing decision-making models in which road's components have to be prioritized for reconstruction such that it optimizes predefined objectives (e.g., Orabi et al. 2009; Lertworawanich 2012; Kaviani et al. 2018; Shiraki et al. 2017). As a tool, optimization modeling is embedded in decision support systems that formulates and solves problems involving (often) multiple conflicting objectives (e.g., Zhu et al. 2019; Xu et al. 2019; Xing 2017). Particularly, in the setting of the transportation network, as a highly critical and intricately interwoven infrastructure, optimized decisions are products of optimizationbased decision modeling that recommend solutions responding to the post-disaster failures of a network's elements (Karlaftis et al. 2007; Zamanifar and Seyedhoseyni 2017). While many reviews investigate the application of optimization modeling in different contexts and fields, it is close to rare in disaster recovery context yet ever-growing essential. This necessity lays on the fact that optimization-based decision modeling, as goes for many complex decision analyses, is an error-prone task that chiefly relies on modelers' judgment (PhillipsWren et al. 2019; Winter et al. 2018; Beven et al. 2015). This inevitable subjectivity could result in committing the error of the third kind to either correctly solve a wrong problem or partly solve the right one (Mitroff and Featheringham 1974). Besides, the criticality and sensitivity of the disaster recovery problem exponentially escalate the consequence of 
errors in DRPTN models. This criticality and sensitivity are due to the cascading impact of disasters in urban areas that propagates on a wide scale and emerges beyond the damage of physical assets to adversely affecting people, economy, environment, and social systems (Kadri et al. 2014). Another reason is the inherent characteristic of disaster recovery problems due to its non-observability as there is sparse matching data of disasters that can be compared with the output of developed DRPTN models for the evaluation purpose (Sargent 1996 and 2011; Day et al. 2009; Kadri et al. 2014). Even if data is partly available, performing a retrospective test to validate a model is a cumbersome task, if not impossible, due to the degree of inconsistency between two datasets of the previous and probable future disasters (Leskens et al. 2014; Celik and Corbacioglu 2010). The non-observability of a problem emphasizes the pressing need of particular care for defining, formulating, and solving prescriptive models that represent critical and sensitive real-life problems. Hence, it is logical to recognize the need for clear identification of possible uncertainty sources and vulnerable parts of DRPTN models that may challenge the validity and quality of the outcome (Buchanan et al. 1998).

To this end, the objective of this paper is to evaluate existing optimization-based DRPTN models' components to identify and discuss the challenges that can provide understanding toward improving the accuracy and practicality of optimization-based DRPTN models. Additionally, since the focus of existing reviews on optimization modeling is mainly on solving algorithms, we performed our analysis based on four phases of optimization modeling as problem definition, problem formulation, problem-solving, and model validation (Nocedal and Wright 1999; Horst and Tuy 1996; Williams 2013). This bottomup approach helps us to review the performance of an optimization model based on its components such as decision factors, choice variables, solving techniques, objective functions, and result analysis methods. Accordingly, the task of problem definition and identifying decision factors is the first phase of optimization decision-making modeling. The second phase is to formulate the problem such that it demonstrates the representative properties of the modeled problem. In this step, analysts set up relations among decision factors and variables and then translate them into mathematical equations. In the problem-solving phase, the selection and implementation of a matching and robust solving approach is the concentration to maintain the quality of the model and the feasibility of outcomes simultaneously. The last phase is to irrespectively verify and validate the model's output such that one can apply it as a reliable solution to a real-life problem. Accordingly, to analyze the identified papers, we first studied the problem definition of DRPTN models to explore the adopted decision factors of models and approaches toward selecting those factors. In the problem formulation phase, we tried to understand as to how relationships among objectives are set and how this relationship can be contextualized for the problem of DRPTN. Furthermore, we investigated the phase of problem-solving in the reviewed DRPTN literature by identifying the rationale for adopting problem-solving algorithms based on objective functions, complexity, and convexity of problems. Finally, we studied the model validation phase to identify approaches toward evaluating the performance of the DRPTN models and the developed mathematical algorithms.

As a review strategy, we began with a systematic search to identify relevant publications based on DRPTN definition and accordingly multiple terms that address hazards, disasters, recovery, roads, and transportation networks. We then used the content analysis approach for analyzing the existing literature. Doing so, we first divided and structured the optimization modeling process into four phases. Afterward, we extracted the information from the reviewed texts that address the adopted phases. Once the representative contents of each publication were categorized, we identified the elements 
of models that were consistent in all publications such as decision factors, solving techniques, number of objective functions, validation efforts. We applied two review questions as (1) What methodological elements of DRPTN models could challenge the validity and contextual application of models' outcomes? (2) How the rationales for structuring the four phases of optimization modeling are conceptually supported? Based on the review questions, we developed several comparing matrixes to state the relation between extracted elements that may lead to possible limitations in the methodologies of DRPTN research. We detected and discussed four research improvement areas as the findings of this study which are: [1] developing conceptual or systematic decision support in the selection of decision factors and problem structuring, [2] integrating recovery problems with traffic management models, [3] avoiding uncertainty due to the type of solving algorithms, and [4] reducing subjectivity in the validation process of disaster recovery models. The identified gaps point out important areas of optimization modeling in the context of disaster recovery that could contribute to the improvement of DRPTN models' performance. The paper presents a contextual understanding of the construction process of DRPTN models which provides insights for decision-makers as to what can be expected from the existing models' performance and what uncertainties they could take into account while receiving decision recommendations of an optimization-based DRPTN model. The study could be also useful for decision analysts and scholars who intend to employ optimization modeling in disaster recovery planning applications.

Within the last decade, a number of review papers addressed several disaster management fields in pre-event and post-event phases such as vulnerability, evacuation planning, emergency response, and reconstruction planning. Among those a few were exclusively devoted to the transportation network and its functionality after disruptive events (e.g., Faturechi and Miller-Hooks 2015; Konstantinidou et al. 2014; Abdelgawad and Abdulhai 2009; Dehghani et al. 2013; Galindo and Batta 2013). However, despite outstanding findings, to the best of our knowledge, addressing the recovery and reconstruction phase of the transportation network was not the focus of those studies. Meanwhile, the literature review on optimization programming is relatively common in different contexts. Existing reviews analyze the optimization methods based on their formulation approach, solving technique, the application of solvers in a specific context (e.g., Fernandes et al. 2018; Wu et al. 2018; Udy et al. 2017; Marler and Arora 2004). While there exist valuable information on the application of optimization solving algorithms, we chose to look at optimization programming as a process. This approach is particularly essential for disaster recovery context because the representativeness of the formulation and reliability of a model's outcome depends on an accurate problem structuring and methodological approach within all four phases of optimization-based decision modeling (Belton and Stewart 2010). Additionally, we failed to identify a review that investigates the adaptability and problem structuring of optimization methods within DRPTN models. Nevertheless, such an effort is of great importance due to the increasing application of optimization programming in the DRPTN context and critical result-sensitive characteristics of such problems.

The remainder of this paper is structured as follows. The next section explains how we approach and analyze the DRPTN literature with respect to the phases of optimization modeling. Section three describes the framework of systematic search and content analysis. Section four and five irrespectively demonstrate the findings and provide related discussions. In the discussion section, we identify the challenges and opportunities within the DRPTN literature based on our interpretation of the findings. Suggestions for future 
directions of DRPTN research and bridging the detected gaps are also incorporated in section five. Finally, the paper provides a summary of the results and a conclusion in section six.

\section{Optimization-based decision-making models and DRPTN}

Optimization is designing or identifying the most favorable choice among a set of alternatives subjected to formalized bounds (Bertsekas 2015). It consists of one or multiple objective functions and a set of variables as well as a defined set of constraints in a finite non-empty subset of a partially ordered space. An optimization model eventually specifies a possible set of non-dominated solutions by varying the selection or order of variables that represent an optimal compromise among objectives (Ehrgott 2005). Variables are alternatives that diverge to optimize objective functions also called choice set or unknowns (Nocedal and Wright 1999). Constraints refer to the applicable limits on decision choices and are responsible for articulating the functional relationships among alternatives. They also allow users to express enforced behavior of a system and indicate certain limitations. A general form of an optimization problem can be shown as $\Phi(k, x)=\left\{\max \left[f_{1}(x), \ldots f_{n}(x)\right] \mid \Phi\left(k^{\prime}, x\right)\right\}$ where $f_{i}(x)$ is the objective function and $\Phi\left(k^{\prime}, x\right)$ is known as the constraints. A multiobjective problem in optimization modeling refers to the notion that the optimal solutions for more than one objective are different and changing the values of the decision vector to improve one objective might result in a decrease in the value of other objectives. Accordingly, Pareto optimality expresses achieving a set of ideal solutions that indicates the optimum trade-off among those conflicting objectives.

For solving an optimization model, problem complexity is an important concept. Problem complexity identifies how difficult it is to achieve the optimal solution for an optimization problem. This difficulty is measurable with the required computational resource that a solving algorithm consumes until it terminates on the optimal or near-optimal solutions. The resource is usually referred to the running time (time complexity) or the used memory (space complexity). When some problems exhibit close asymptotic behavior in consuming computational resources for obtaining optimal solutions, then they shape a class of complexity. Insights from the computational complexity of problems especially tackling non-convex problems can locate the cumbersome part of the formulation, which indicates where it is possible to aggregate, decompose, or simplify and helps to model the problem effectively (Tovey 2002).

As an inherent property for some classes of optimization problems, every local optima is a global optima. These problems are referred to as convex optimization problems (Bertsekas 2015). Informally, convexity in optimization means that objective functions and feasible sets formed by constraints shape a convex feasible region that ensures the existence of the global minimum. Convexity analysis refers to the evaluation of the geometric feature of the feasible region toward constructing smooth convex objective and constraints functions. Detecting the convexity of the feasible region of the problem provides useful insights to assimilate the complexity of the problem and eventually selecting an efficient solving algorithm (Johannes 2011). As Fig. 1 illustrates, presuming that the right problem is recognized, we present the optimization modeling as a process with four main phases namely definition, formulation, solving, and validation. The following section introduces the properties of each phase and its importance in the context of DRPTN. 


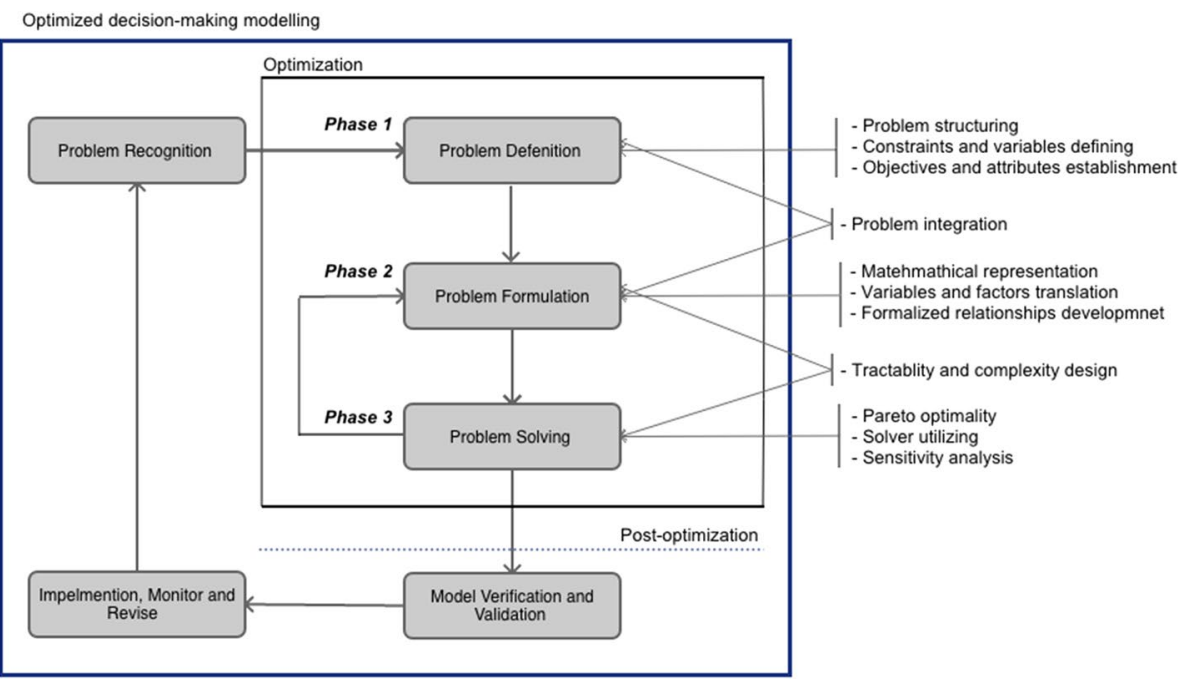

Fig. 1 Phases of decision-making modeling and steps of optimization programming

\subsection{Problem definition}

Modeling of a real-scale problem aims at abstracting the perceived system and the problem in one environment (Phillips 1984). It initiates with identifying the problem and selecting its decision parameters as part of problem structuring (Keeney 1992). In optimizationbased decision-making modeling, the problem's components are the decision factors, which express the system's function, and decision variables that control the behavior of decision parameters. Among decision factors, attributes evaluate the performance of the system and the distance to the desired state of the system articulated by objectives. Though decision-making models eventually optimize the given objective functions, quality of the resulting outcome depends on 'the completeness of the model in representing the real system' (Taha, 2007). Meanwhile, representing the real system is highly contingent upon defining decision factors such as objectives and attributes (Belton and Stewart 2012; Keeney and Gregory 2005).

There are many well-posed optimization problems with clearly defined decision parameters such as production efficiency problem, manufacturing problems, blending problems (Zopounidis and Doumpos 2002). However, in the disaster recovery planning context, problems do not often emerge clearly labeled or with fully defined properties. In the aftermath of a disaster, objectives and preferences are dynamic and hardly recognizable (Leskens et al. 2014; NRC 1999). Additionally, effective attributes and even in some cases alternatives are vague as well. Hence, a thorough investigation of the problem definition step as the preliminary phase of the optimization modeling process is vital. Even more so in the context-dependent and critical problem of DRPTN that is highly complex and cannot afford conceptual error in representing the real system due to a broad impact of results on multiple accepts of a big scale society. On this ground, we study the variety of attributes that DRPTN studies developed and analyze the rationale for choosing those attributes. 


\subsection{Problem formulation}

This phase formulates a mathematical translation of the defined problem and establishes sets of relationships among variables and decision factors (Morris 1967). When modelers achieved an equitable set of decision factors in the problem definition phase, in this phase they seek the desired arrangement among them (French 2018; Williams 2013). Additionally, selecting the target set of variables of the problem is a task of this step since those variables are part of possible solutions that ultimately shape the feasible region of the optimization problems (Ehrgott 2005; Lange 2013). Although this step has many interrelations with the phase of problem definition, since both are parts of the problem structuring, yet it cannot proceed unless the outcome of the first step is available. Unlike problem definition during which modelers select decision parameters, in the problem formulation phase, they decide as to how to treat decision parameters. Problem formulation, additionally, deals with integrating the target variable sets and assign values to objective functions to achieve a meaningful and formalized mathematical expression of the intended problem.

In doing so, traffic assignment simulation is a common sub-model for assigning value to traffic decision factors of optimization models in transportation planning. Traffic assignment models simulate the traffic on a network based on origin-destination travel demand to identify the traffic flows distribution on links on which equilibrium is obtained. There are two general approaches for traffic assignment; System Optimum and User Equilibrium (Wardrop 1952). The System Optimum traffic assignment approach assigns the traffic flow to links in order to optimize the ideal possible traffic distribution on the whole network. In contrast, User Equilibrium distributes the traffic flow on the network to reach equilibrium on links based on the utility of routes and the assumption of rationality of drivers (Wardrop 1952). DRPTN optimization problems mainly design the order of variables to optimize the value of traffic assignment models next to other objectives.

In planning for a transportation network, physical assets such as bridges, highways, or links are common choice variables. Administrative or non-physical components are also variables that either represent or impact the performance of the transportation network such as traffic calming strategies, rerouting plans, options of lane management, and travel demand regulations. Problem formulation, in DRPTN, usually adopts the physical components as a variable set to prioritize the recovery tasks of those components that optimize an objective or the trade-off between multiple objectives. These objectives represent different problems after a disaster such as relief distribution, resource allocation, or network design problem. Basically, each problem is associated with its specific choice set variables, which are configured next to the transportation network's components. For example, relief distribution problem adds relief units to the variable of the optimization model or the problem of resource allocation incorporates available work teams and budget into the computation. The integration between those problems eventually constitutes the final variable set as well as the configuration among them to compute the objectives. This integration in DRPTN is critical because, on one hand, the statement of an optimization problem is affected by the nature of relations among decision parameters. On the other hand, a practical multifacet problem of DRPTN needs to address the goal of modeling by incorporating effective objectives. This motivated us to investigate the problem formulation phase to understand variables and the problem integration within DRPTN models. 


\subsection{Problem-solving}

Solving an optimization problem could be the simplest step of optimization modeling because it entails the use of well-defined optimization algorithms and tools (Taha 2007). Nevertheless, selecting an efficient, robust, and fitting technique that promises a reliable optimal solution is a challenging part of solving DRPTN problems. In that context, deterministic and non-deterministic algorithms are the main approaches toward finding solutions for optimization problems. Deterministic algorithms return exact minima points of the solution space. Examples of these algorithms are; Sequential Quadratic Programming, Generalized Reduced, Gradient, and Dynamic Programming. Non-deterministic approaches are heuristic and meta-heuristic population search, evolutionary or trajectory search, and their extensions that lead to methods such as Genetic Algorithms, Simulation Annealing, Particle Swarm, Harmony search, and Tabu Search (for a review see e.g., Blum and Roli 2003). These algorithms provide feasible but not necessarily optimum solutions and cannot submit a mathematical proof of whether the returned configuration is minimal or at least how good it is compared to the optimum solution (Schneider and Kjrkpatric 2006; Talbi et al. 2012). Having that in mind, when the degree of complexity and size of a problem increases, deterministic algorithms consume an unreasonable amount of computational resources. It means solving a big-size non-convex NP-hard problem in polynomial time would be extremely difficult (unless $\mathrm{NP}=\mathrm{P}$ ). In this case, employing a non-deterministic method is a logical choice that relatively easily handles such a problem with the effort that grows polynomially as do the size of the problem.

Although the mathematical procedure of solving DRPTN problems with non-deterministic methods is generally correct, the validity of a solvers' outcome cannot be properly examined (Festa 2014; Rardin and Uzsoy 2011) as it operates as a black-box solver and without any further problem-specific adjustments (Rothlauf 2011). Context-independent, general-purpose, or black-box solvers cannot explore the structural properties of the objective function. A feature of these algorithms is that the outcome solution might be inferior to purpose-specific algorithms that solve the same problem (Marti and Reinelt 2011).

This is a major concern when researchers develop sophisticated algorithms to solve mathematically modeled DRPTN problems while the rationale behind the selection of the optimization methods remains unevaluated, especially in the sensitive problem of DRPTN that exact result is vital for reliable planning engaging with human life. Therefore, in the context of DRPTN uncertainty due to the utilizing solving algorithm can be a challenge when multiple objective functions are involved in the modeled problem (Liefooghe 2011; Horst and Tuy 1996; Talbi et al. 2012). Particularly, uncertainties and biases due to the quantification of values or assigning preferences (e.g., in priori decomposition-based approaches) also question the validity of solutions since epistemic uncertainty can easily propagate to the optimization output (Limbourg 2005) even when the model is mathematically correct. Therefore, it is logical to investigate the impact of objective level in DRPTN models on the accuracy of results and rationale behind utilizing solving algorithms in DRPTN models. In this study, the objective level refers to the number of objective functions that a model intends to optimize. Single-objective optimization problems contain one objective function and the bi-objective level problem refers to problems with two objective functions that usually formulate a leader-follower game. Similarly, models that are formulated with three and four objective functions are identified as multi-objective. When a model seeks to optimize more than four objective functions, it forms a many-objective problem. 


\subsection{Model verification and validation}

Model validation and verification are two concepts toward irrespectively evaluating the reliability of a model's outcome and quality of the solution. Model validity indicates how well the optimal solution of the model is to solve the real-life instance of the intended problem. Model verification, however, demonstrates how well the output represents intended developed mathematical relationships among parameters (Oberkampf et al. 2003; Oberkampf and Roy 2010; Sargent 2011). Validation is a process that attempts for obtaining sufficient confidence (if there can be any) that the solution of the model can be considered valid for its intended application. The classical approach of validation is based on comparing the outcome of the model with the known experimental measurement of the same problem in reality when the input set for both systems are equivalent (Roy and Oberkampf 2011; Sargent 1996).

Validation in the context of DRPTN is challenging because the confidence threshold in models is set relatively high since DRPTN-related decisions are associated with human life and enormous socioeconomic losses. Additionally, disaster recovery problems are highly prone to epistemic and aleatoric uncertainties as well as parametric errors due to the complexity, context-dependency, and time-stretched process of the decision-making. Therefore, the "value of model to user" dictates the demand for maximized model confidence (Sargent 2011). Existing optimization models for disaster recovery problems deliver fast and efficient solutions while they might be limited in representing many of the crucial realities of the modeled system. In such a situation, the validation of models is an imperative phase of the modeling process to evaluate the quality of the model. It answers the question of whether the result can be trusted as bases for making decisions in a critical engineering socioeconomic situation (Babuška et al. 2007). To provide a better understanding of the significance of this question we investigate the validation and verification efforts within DRPTN models.

\section{Review and analysis methodology}

\subsection{Search strategy}

We performed a systematic literature search to find optimization studies that addressed disaster recovery planning for damaged transportation networks. Based on our earlier definition of DRPTN, we established certain exclusion and inclusion criteria to design clear boundaries for the literature search. The four main criteria were, first, the candidate publication studies a component of a transportation network. Second, the system disruption of the study occurred due to a hazard or a large-scale disruptive event. Third, the target of papers is to present a recovery, reconstruction, or repair planning for damaged elements. Fourth, studies use optimization modeling to develop the problem. The search task was according to various terms addressing disaster and transportation network in the abstract, title, and keywords of the publications. We repeated the search task with different terms representing the same concept. For example, addressing the term disaster, we performed the search with terms such as "disaster", "hazard","extreme event", "earthquake", "landslide", "emergency" "flood", and "tsunami". Similarly, for the transportation network, we searched for "transportation", "traffic", "bridge", "link", "road", "highway", and "network". Thereafter, 
we discarded duplicate publications as the outcome of multi-platform searching and sifted the searching process by limiting disciplines as well as the exclusion of irrelevant keywords. Selected disciplines were business, management and accounting, computer science, decision sciences, earth and planetary sciences, engineering, environmental science, mathematics, and multidisciplinary.

Reducing errors in finding related publications, we tried to strictly follow our designed benchmarks. Additionally, we agreed to not use multiple screening or filtering steps presented by the used platforms. Instead, we chose to manually investigate the final set of references $(n=910)$ based on three steps of content analysis to learn whether the publications belong to the scope of our study or not. These steps were irrespectively content analysis of (a) abstract, (b) abstract, methodology or problem description section and c) full-text of the publications $(n=241)$. Moreover, we also used the snowball method by performing a forward referencing search in the selected papers' reference lists that has led us to identify three additional publications.

\subsection{Content analysis}

The content analysis is based on analyzing the methodology of DRPTN studies following the phases presented in Sects. 2.1-2.4. We evaluated the studies with respect to possible sources of uncertainties and conceptual vulnerabilities in the formulation, problem structuring, problem-solving, and validation process of DRPTN decision-making models. For this purpose, we performed a directed content analysis and measured the number of studies

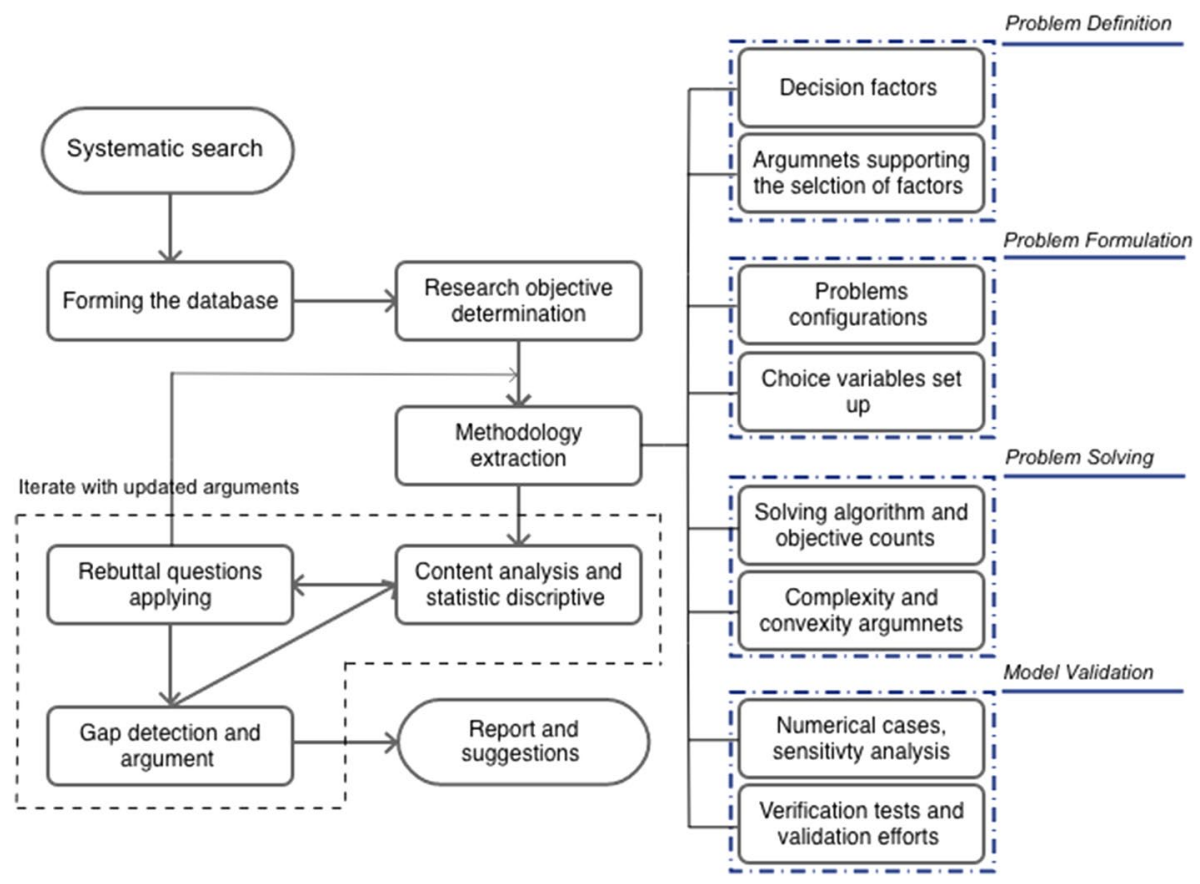

Fig. 2 The systematic review and gap analysis flowchart for the literature of DRPTN 
for all extracted information based on Fig. 2. We framed two review questions to approach the publications as (1) What methodological elements of DRPTN models could challenge the validity of models' outcomes? (2) How the rationales for structuring the four phases of optimization modeling are conceptually supported? Fig. 2 shows the detailed steps of the content analysis framework that we describe in the rest of this subsection.

In the first step, we extracted the elements of the methods applied to our content analysis strategy and the framework of optimization decision-making process introduced in Sect. 2. Doing so, we analyzed various components of methodologies such as decision attributes, formulation approaches, solving methods, convexity and computational complexity analysis, arguments supporting the selection of solving methods and the selection of attributes as well as model validity and verification arguments. The second step focused on performing multiple identification and grouping of optimization components including objective counts, attribute types, employed traffic performance metrics, problem integration types, types of solving algorithms, and types of variable sets. For example, we categorized the attributes within three main classes of emergency, traffic, and economic. Accordingly, the traffic factors include attributes that represent the performance of the transportation network such as travel time, capacity, or density. Emergency factors are attributes that respond to the social and individual urgent needs after disasters and demonstrate the performance of emergency response operations such as relief distribution, housing, or population. Lastly, economic factors represent the budget and cost-related attributes incorporated in the planning such as direct or indirect damage costs.

After that, we developed several comparing matrixes to state the relation between extracted elements that may lead to possible limitations in methodologies of DRPTN research as: numbers of objectives and used solving methods, complexity class arguments and used non-deterministic algorithms, formulation approaches and integrated problems, as well as traffic engineering methods and the application of the post-disaster travel demand. Also, the analysis included the corresponding presented theories and methodologies for establishing attributes, selecting solving algorithms, and developing validation approaches in each study. Finally, we analyzed the frequency of the detected gaps to report challenges that are overall in the body of DRPTN literature. Accordingly, the next section presents the results of performing content analysis in the reviewed DRPTN literature.

\section{Findings}

This section demonstrates the findings of the content analysis in each phase of DRPTN optimization modeling process.

\subsection{Problem definition}

Figure 3 shows the attributes that DRPTN models employ. Additionally, Table 1 demonstrates the types of attributes and their combinations that are categorized according to whether they focused on emergency, traffic, or economic goals.

Figure 3 and Table 1 show that most DRPTN studies establish traffic attributes to measure the technical performance of networks such as mobility and level of service. In some cases, a combination of traffic attributes represents an attribute for network functionality. Figure 3 shows that Travel time is the most frequent attribute to measure the quality of the traffic service after disasters and Travel flow appears in $41 \%$ of the studies. 


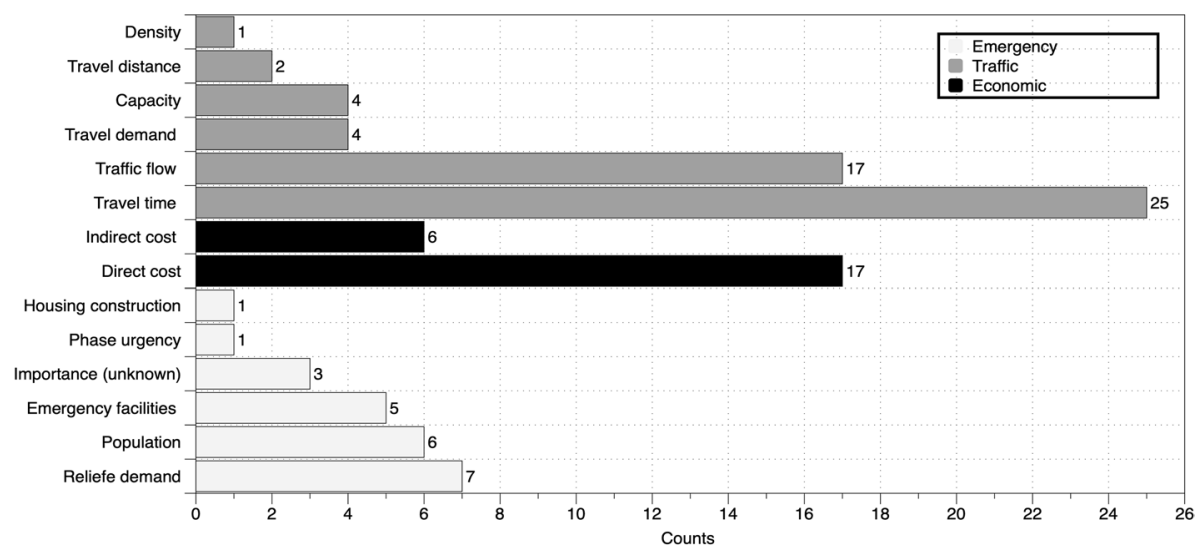

Fig. 3 Attributes employed in DRPTN as well as their categories

Table 1 Amount and share of attributes in three categories as well as their combination within DRPTN studies (Em: Emergency, Tr: Traffic and Ec: Economic factors)

\begin{tabular}{llllclcc}
\hline Factors & $\mathrm{Tr}$ & $\mathrm{Ec}$ & $\mathrm{Em}$ & $\mathrm{Em} / \mathrm{Tr} / \mathrm{Ec}$ & $\mathrm{Em} / \mathrm{Ec}$ & $\mathrm{Em} / \mathrm{Tr}$ & $\mathrm{Ec} / \mathrm{Tr}$ \\
\hline Counts & 39 & 19 & 16 & 5 & 1 & 7 & 9 \\
Share $(\%)$ & 97.5 & 47.5 & 40 & 12.5 & 2.5 & 17.5 & 22.5 \\
\hline
\end{tabular}

Furthermore, two studies (5\%) adopt Travel distance and five studies (10\%) incorporate Travel demand and link Capacity to measure the achievements of their objectives. With respect to economy attributes, in the whole, 19 studies (47.5\%) consider budget-related attributes such as Direct cost, which simply refers to the repair cost of transportation components. Six publications (15\%) additionally apply Indirect cost which in four studies was associated with the direct cost. Indirect cost represents the economic disruption due to network failure or secondary costs due to the travel delay. In total, nine studies $(22.5 \%)$ combine economic and traffic attributes in their models.

Emergency attributes address critical civil needs after disasters or represent metrics that can influence the risk of fatality. For example, Relief demand as a major attribute in this category in seven studies $(17.5 \%)$ refers to traffic nodes to which emergency supply should be distributed. Five studies (12.5\%) incorporate the attribute of Emergency facilities for links that provide access to those nodes. Six studies (15\%) consider Population in a traffic zone or Population that is served by links to addresses an emergency aspect of the post-disaster situation. Furthermore, 16 studies introduced emergency attributes and five studies $(12.5 \%)$ incorporate attributes to measure the social impact of disasters on an urban area. Finally, based on Table 1, five papers (12.5\%) develop the attribute sets with all three categories of decision factors.

Lastly, we analyze the content of DRPTN studies to identify information or approaches that support the selection of attributes. In this regard, $22.5 \%$ of the studies provide conceptual arguments to theoretically support this selection or identification. For instance, Feng and Wang (2003) provide a section to identify objectives of 
Table 2 The condition of variables as the alternatives of optimization models within DRPTN

\begin{tabular}{lccl}
\hline Variables & Count & $(\%)$ & Note \\
\hline Components & 15 & 37.5 & Bridge, railway, link. route, segment, node \\
Components and resources & 11 & 27.5 & Integration of two sets of alternatives \\
Sequence of recovery of components & 7 & 17.5 & Including sets that are combined with resource \\
& & & choices \\
Set of components & 3 & 7.5 & Strategic or zone-based solution set \\
Sequence of components and resources & 3 & 7.5 & Links and contractors/ work troops \\
Sequence of assigning resources & 1 & 2.5 & Relief units \\
\hline
\end{tabular}

Table 3 Integration of post-event problems with the recovery

\begin{tabular}{lcl}
\hline Task & Count & $(\%)$ \\
\hline Recovery and network design & 11 & 27.5 \\
Recovery and task scheduling & 9 & 22.5 \\
Recovery and resource allocation & 8 & 20 \\
Recovery & 8 & 20 \\
Recovery and relief distribution & 4 & 10 \\
\hline
\end{tabular}

the planning, recovery characters, resource constraints, and decision-making process to accordingly justify the selection of the attributes. In another case, performance attributes by Unal and Warn (2015) “... were selected to be representative and to facilitate the restoration design based on available data and reasonable computational efforts" and was supported by specific details for each parameter and importance of the selection.

\subsection{Problem formulation}

To investigate the formulation approach of DRPTN optimization problems we analyzed the integration of objectives, sets of choice variables as well as objective value assignment approaches in traffic flow distribution models. Table 2 shows the choice variable sets of optimization DRPTN models.

Within the transportation network's components, DRPTN models regard bridges, railways, routes, segments, links, and nodes as variables. These physical components form the alternative set of $97.5 \%$ of models, of which 25 studies (63\%) integrated transportation network's components with other variables. DRPTN models identify resources such as budgets, work troops, and contractors, but always in combination with physical components (except for one study that uses resources independently). 11 studies (27.5\%) focus on sequences of alternatives to optimize recovery activities with respect to all possible orders among alternatives. Furthermore, three studies (7.5\%) adopt sets of components as the variables defined by selected recovery strategies or a network zone. As the second part of the findings, Table 3 shows the integration of objectives and consequently sub-problems within DRPTN.

Four studies (10\%) formulate a model of relief distribution and recovery problem with integrating variables and objectives of both problems. This problem integration prioritizes the recovery tasks that timely meet the post-event needs or optimizes the aid distribution 
process by solving a network routing problem. Nine studies $(22.5 \%)$ formulate tasks of scheduling and recovery problems in one optimization model to assign the recovery tasks to contractors and optimize the traffic performance of a network against cost or duration of the recovery. This formulation also optimizes multiple metrics of the network subjected to scheduling constraints such as material or machinery limitations. Resource allocation and recovery problem integration ( 8 studies, $20 \%$ ) optimizes the sequence of recovery activities for minimizing the budget or reconstruction duration while maximizing a technical metric of the network. Also, some models assign resources to a sequence of recovery projects in which a compromise between technical objectives of the network and reconstruction cost can be found. 11 studies (27.5\%) formulate the integration of network design problems and recovery problems to prioritize recovery tasks according to the network traffic load. These studies propose the use of traffic assignment on a degraded network to identify the importance of specific links. In addition, the network design problem can indicate the optimized recovery order that reduces the travel time for emergency vehicles.

Finally, 23 studies (57.5\%) formulate the decision models based on the output of traffic assignment models that assign quantified value to the objective functions. Therefore, we investigated the traffic assignment approach within DRPTN models, to understand how DRPTN models are formulated to address post-disaster travel demand of the network. Accordingly, two studies (5\%) modify the regular travel demand for the post-disaster condition addressing limitations in the functionality and accessibility of the network. Additionally, except for one paper that considers the System Optimum approach, User Equilibrium traffic assignment is the dominant approach for assigning traffic flow to the network.

\subsection{Problem-solving}

$75 \%$ of the DRPTN models (30 studies) use non-deterministic algorithms in the problemsolving phase such as genetic algorithm, simulated annealing, tabu search, and ant colony. To understand the rationale of selecting non-deterministic algorithms and the impact of this selection on the quality of outcomes we investigate the objective level of optimization problems that are solved by non-deterministic methods. Additionally, we analyze the arguments that support selecting non-deterministic methods to solve the optimization problems. Figure 4 shows the relation between objective numbers and the rate of studies that used non-deterministic methods for solving the intended problems in each class of objective count.

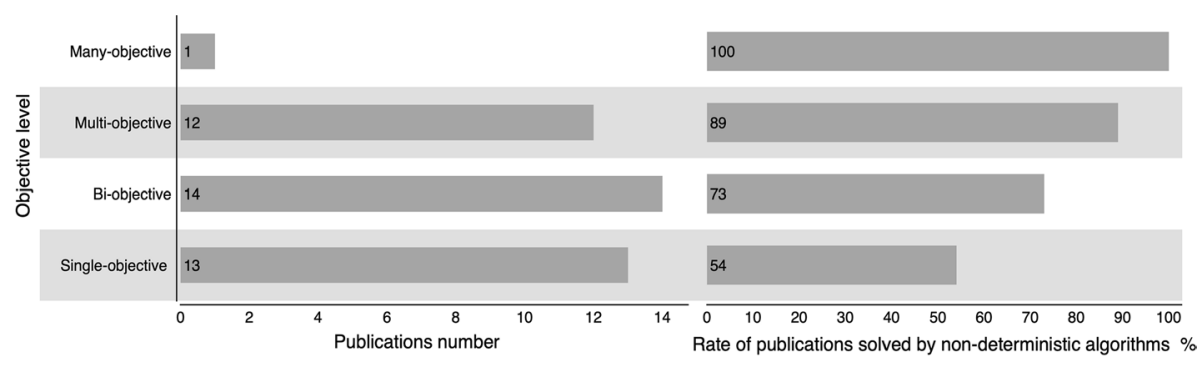

Fig. 4 The use of single, bi, multi, and many objectives formulation in DRPTN research (left) and percentage of used non-deterministic methods in each objective number level 
Table 4 Description and amount of discussions over applying non-deterministic algorithms in optimization problems

\begin{tabular}{lll}
\hline Count & $\%$ & The argument for utilizing the non-deterministic methods \\
\hline 15 & 50 & No discussion presented \\
7 & 23.3 & NP-hard according to characteristics discussed by other sources \\
5 & 16.7 & Due to computation cost \\
2 & 6.7 & Computation complexity discussed \\
1 & 3.3 & Avoiding Braess's paradox (Braess 1968) \\
\hline
\end{tabular}

Non-deterministic algorithms solved $54 \%$ of the problems with one defined objective function. Similarly, $73 \%$ of the problems with two and $89 \%$ of the problems with three and four objectives are solved by non-deterministic methods. Figure 4 also shows that problems with one and two objective functions reach $67.5 \%$ of the whole DRPTN optimization models and one study developed the optimization model with more than five objective functions. Furthermore, Table 4 provides an overview of the rationale that DRPTN studies reported for choosing non-deterministic optimization methods.

Nine papers (30\%) address the complexity of the problem. Two of those studies $(6.7 \%)$ fully discuss the class of complexity of their optimization problems and seven studies (23.3\%) identify a known Hard problem within the original problem which results in an NP-Hard or Complete problem thus accordingly derive the methodology toward utilizing non-deterministic methods. Furthermore, three publications (10\%) point out the convexity state of their problem although without a report of an investigation over visualized geometric of the search space or computing the Hessian matrix for the second-rate derivative of the objective function. Additionally, five studies (16.7\%) mention the computational cost of solving methods as the reason for selecting non-deterministic algorithms, although no representative computational indication could be identified.

Table 5 Efforts and arguments toward verifying and validating DRPTN models

\begin{tabular}{lcc}
\hline Arguments on validation of the models & & \\
\hline Case & Count & $\%$ \\
\hline Numerical case for validation & 8 & 20 \\
Validation is left for future studies & 4 & 10 \\
No specific argument is provided & 28 & 70 \\
\hline
\end{tabular}

Table 6 Efforts toward verifying solving algorithms of DRPTN models

\begin{tabular}{lcl}
\hline Efforts on evaluating the performance of the algorithms & \\
\hline Case & Count & $\%$ \\
\hline Numerical example & 10 & 25 \\
Sensitivity analysis & 11 & 27.5 \\
Algorithms computational performance & 18 & 45 \\
Algorithms verification tests & 8 & 20 \\
No effort identified & 11 & 27.5 \\
\hline
\end{tabular}




\subsection{Model validation}

In the validation phase of the optimization decision-making models, some DRPTN studies provide approaches and arguments to assess the quality of algorithms and models while in the majority of cases we failed to identify explicit argument on the validation phase of the developed models. Tables 5 and 6 demonstrate how reviewed DRPTN studies evaluate the performance of algorithms and validate the solution of models and to what extend studies did not offer a direct argument referring to the validation phase of DRPTN models.

Results show that eight publications (20\%) represent their numerical examples as a validation approach for the developed model. Also, ten studies (25\%) provide numerical examples to conclude the performance or quality of the developed algorithm. For example, a study state that the reason for providing a numerical example is 'to verify the feasibility and applicability of the method' and claims are made that [...] it also indicates that this method is clear, efficient and adaptive and it can provide theoretical foundation and technical evaluation (Yuan et al. 2014). Another case highlights that the numerical example '... proves the validity of models and algorithms, provided a scientific foundation for the government to make reasonable rush-repair scheduling when the disasters occur' (Zhang and Lu 2011). On the other hand, some studies directly point out that the presented application example '...is to illustrate the use of programming formulation...' (Orabi et al. 2010) or to only '...evaluate the algorithm's performance...' (Wang et al. 2011) within the model. For example, Sato and Ichii (1995) present a numerical example to test the efficiency of the solving algorithm and Duque and Sörensen (2011), El-Anwar (2016a, b), or Hackl et al. (2018) emphasize that experimental future works are required for validation of the model.

Furthermore, 18 studies (45\%) evaluate the algorithm's computational performance. Additionally, eight studies (20\%) employ standard verification tests to evaluate the mathematical performance of their models such as consistency tests, simplified testing, output comparison with similar models, and comparison with all permutated results (in small size problems). Finally, 11 publications (27.5\%) analyze the sensitivity of variables and weight vectors aiming at assessing the performance of models.

\section{Discussions and suggestions}

Based on the findings of the previous section, we provide arguments for the identified challenges and opportunities within each phase of the DRPTN optimization modelling process.

\subsection{Problem defination}

The broad set of attributes within DRPTN offers divers and exhaustive representations of the real system which itself is diverse and stochastic. At this stage, practice can benefit from various problem definitions DRPTN literature provides to address different and specific real-world problems. Equally important, DRPTN models have the potential for improvement in enhancing the completeness of the attributes set. One of the reasons is the absence of highly effective attributes in the decision factor sets of some DRPTN models. For instance, although the transportation network disaster recovery is a technical problem, it serves a social system (Nigg 1995; Lubashevskiy et al. 2017). Nonetheless, only five studies incorporate social vulnerability or an indicator that measures the social impact of recovery operations. Additionally, the expected outcome of a disaster recovery model in 
practice is to alleviate the calamitous impact of disasters on societies given its sociotechnical aspects. Nevertheless, the main goal of DRPTN studies is set to improve only the technical performance of the road network, since $95 \%$ of studies incorporate traffic attributes and $50 \%$ of them introduced their model only based on traffic attributes. Furthermore, only five sources (12.5\%) include all three clusters of decision factors and seven studies (17.5\%) introduced a combination of traffic and emergency factors in their formulation.

Additionally, the interaction of a transportation network's components with other critical infrastructure networks (lifelines) is a widely acknowledged critical decision factor in disaster management (Zhang 1992; Cavalieri et al. 2012; Kadri et al. 2014). Nevertheless, we could not find this factor in any of the reviewed studies as an attribute toward optimizing recovery activities. Lifeline interaction is an important attribute for prioritizing the recovery of links since early-stage damage control in other interconnected infrastructures such as the gas delivery network or power lines is essential to avoid secondary, technical, and cascading hazards. Similarly, only $12.5 \%$ of the studies included the level of access to critical facilities, which shows that "accessibility" and, in particular, access restoration to service providing nodes such as hospitals, fire stations, strategic points, control centers, or shelters have not been considered sufficiently yet.

A worth noting finding is that in the majority of the reviewed DRPTN studies (31 studies, $77.5 \%$ ), we could not identify a systematic approach or a conceptual argument to support the incorporation of attributes in the developed decision-making models. This argument is also consistent with the identified challenge by some studies in different fields (Ha and Yang 2018; Tiesmeier 2016; Fekete 2019). However, we are not able to pinpoint the cause, yet, the absence of incorporation of highly effective attributes such as accessibility and social factors within the attribute set of DRPTN models might be the result of the absence of a formal or informal effort to identify effective decision factors.

To control subjectivity and reduce conceptual errors in establishing decision factors, we suggest the development of a systematic framework toward the selection of decision factors in the DRPTN context. To avoid the error of the third kind, it is critically important that such efforts recognize the collectivity of the disaster recovery problem. It is a necessary task that future studies address the identification of complete and collective sets of decision factors or establish accredited evaluation criteria for such a set. Accordingly, a broad descriptive and qualitative analysis of the problem in the initial steps of research and dedicating more time and effort into the problem conceptualization and problem structuring is inevitable.

\subsection{Problem formulation}

DRPTN studies addressed essential post-disaster problems by integrating different objectives in one decision environment such as relief distribution, route planning, and resource allocation. As a whole, DRPTN studies cover many variables of the post-disaster setting. Meanwhile, DRPTN models formulate representative properties of the post-event network performance regardless of administrative variables. Additionally, the problem formulation of DRPTN models might be challenging in terms of contributing to post-disaster traffic quality in surviving networks with the recovery schemes. This interpretation is apparent based on Tables 2 and 3, as we could not identify studies that integrate disaster recovery planning and traffic management problems. Nor could we identify a study that adopts traffic management measures such as redistribution of the traffic flow, rerouting, signals 
management, lane reversal, temporary shoulder capacity, etc., as an administrative variable set of the DRPTN optimization problem.

Regarding the representativeness of DRPTN models in the formulation phase, the status quo of DRPTN studies is using the assumption that post-event traffic flow follows a sub-pattern of the existing pre-event traffic flow along some degree of network geometric restrictions (damaged nods or links). This assumption is understandable due to the high degree of uncertainty and complexity in predicting the route choice of travelers after a disaster that within DRPTN exists a lack of interest in estimating the post-disaster traffic condition of networks since User Equilibrium is the most popular approach within DRPTN models. However, this might be a too simplified assumption since, on one hand, User Equilibrium philosophy is based on the reflection of the optimal state of each traveler according to his or her perception in a normal condition and perfect information environment. In addition, according to Braess's paradox (Braess 1968), the equilibrium is not necessarily relaxing in the ideal state of the network. On the other hand, in the significant information lack condition of the post-disaster environment (Day et al. 2009), the route choice utility (Dobler 2011), serviceability of the system (Chang and Nojima 2001) and even users of the network (Iida et al. 2000) are radically different from the pre-event condition. On this ground, User Equilibrium cannot realistically represent many features of traffic flow in the post-event distributed network since several fundamental assumptions of this approach are violated in the post-disaster traffic behaviors. Accordingly, the findings suggest the challenge of formulating DRPTN models in assigning representative values to objective functions as well as integrate traffic management variables with recovery options.

To improve the DRPTN formulation, applying the User Equilibrium approach for the post-disaster phase can be revised by manipulating variables and the problem integration. Doing so, we suggest shifting the role of traffic assignment from a post-event unknown variable to a known target value, i.e., design the optimization problem such that the model finds the optimized order of variables to reach the ideal given state of the network in the Service Optimum approach. This formulation also entails including traffic management measures as an auxiliary alternative set next to the recovery activities. Using integrated traffic management and recovery planning, planners can assist and direct the users' route choice in the post-event phase. This formulation approach optimizes travel demand of the ideal traffic flow distribution by designing a new network plan based on the surviving network, recovery options, and updated administrative regulations (e.g., lane reversal, demand regulation signal management). It can indicate how external interventions by planners after a disaster (recovery of links and traffic management) lead the network toward reaching the optimum equilibrium based on the Service Optimum traffic assignment approach.

\subsection{Problem-solving}

The incorporation of non-deterministic algorithms within the problem of DRPTN in many cases overcomes the challenge of solvability of DRPTN problems. In fact, DRPTN studies could very effectively harness the advantages that non-deterministic methods offer. Therefore, it is impossible to ignore the benefits of fast and feasible solutions of non-deterministic algorithms, however, results also suggest the challenge of conceptual and computational support for selecting the solving method as well as the absence of complexity and convexity analysis before choosing the algorithms. Figure 4 shows the increase in employing nondeterministic algorithms when the number of objectives rises. Accordingly, a compromise between certainty and effectivity is apparent within DRPTN models. The more objectives 
do models incorporate, the less certain the final solution is. On the contrary, the more solving algorithms try to yield an accurate mathematical outcome; the model can cover fewer objectives. Thus, it might exhibit a lack of inclusion to address various aspects of a post-disaster condition. The compromise between certainty and effectivity arises since; (a) subjectivity and errors within the process of selection and quantification of decision parameters and (b) the urge for use of non-deterministic algorithms due to the complexity of a problem, both cardinally grow with the number of objectives (Vianna and Vianna 2013; Limbourg 2005). This is a challenge for the quality of multi-objective optimizations when a result-sensitive context-dependent problem is solved with a context-independent method with no guarantee of returning optimal results at the global level (Ishibuchi et al., 2008). This challenge is highlighted when $73 \%$ of bi-objective and $54 \%$ of single-objective problems have been solved by non-deterministic algorithms (Fig. 4) even though the exact methods are generally valid for single and bi-objective optimization problems up to a large size (Vianna and Vianna 2013; Liefooghe 2011). Consequently, although the increase in objective numbers provides a more contextually exhaustive and effective model to cover different dimensions of a disaster recovery problem, it also comes at its impact on the certainty of the algorithm's solution. Therefore, given the critical engineering socioeconomic nature of DRPTN problems, the right balance between exactness of result and inclusion of the model is a critical consideration that might require broader attention to the problemsolving phase of DRPTN optimization modeling process. To reduce uncertainty in solving disaster recovery problems, it perhaps would make more sense to utilize non-deterministic methods only for optimization problems with multiple objectives and constraints that even their approximation apt to be a cumbersome task.

Besides the absence of convexity analysis, it is believed that 'the complexity class of an optimization model dictates the nature of the solving method' (Taha 2007). Yet, 53.3\% of studies chose the solving method regardless of complexity investigation of the problems and only two studies present a detailed discussion on complexity analysis of the problems. Moreover, although it is commonly understood that when a problem is NP-Hard then nondeterministic methods are the method of choice, the fact is ignored that many NP-Hard problems can be still solved relatively fast with standard mathematical methods (Rothlauf 2011). Therefore, it is logical to consider both complexity class and convexity analysis of DRPTN problems before choosing the solving algorithm since when an optimization problem formulates a convex problem, it is very likely solvable deterministically and efficiently (Boyd and Vandenberghe 2004; Grötschel and Holland 1991). On this ground, while solving a critical result-sensitive problem of DRPTN, an important consideration is that approximation is secondary to the deterministic approach as long as an exact solution is achievable. However, complexity class and convexity analysis of the DRPTN models have not been sufficiently emphasized. Among $50 \%$ of the reviewed studies, we failed to spot conceptual or computational justification that supports the application of non-deterministic algorithms for a specific problem, even though the size of the problem, in most of the cases, was relatively small and the number of objectives in $67.5 \%$ of cases was not exceeding two. Therefore, we suggest that future research evaluate the complexity class and convexity state of the problems of interest before choosing a solving method, As Rockafellar (1997) highlighted, 'The familiar division between linearity and nonlinearity is less important in optimization than the one between convexity and non-convexity' (Rockafellar 1997). Another suggestion is to consider formulation approaches which likely form a convex solution space such that exact methods can solve the problem. As an instance, we can highlight the work of El-Anwar et al. (2016a, b) which formulates a mix-integer optimization 
problem with a convex cone in the DRPTN context and could efficiently improve the nearoptimal solution to the optimal solution.

\subsection{Model validation}

Despite the non-observability of DRPTN problem, the fact that $72.5 \%$ of studies conducted efforts to systematically evaluate the outcome of the models indicates that within the field of DRPTN the awareness is established that the DRPTN problem is highly result-sensitive thus cannot afford inaccuracy in the solution and requires a relatively high level of confidence. At the same time, results point out a potential improvement area in validating disaster recovery models. That is because, based on the classical definition of the model validation, (which relies on the comparison of a known solution to the model's outcome), efforts toward validation of DRPTN model are limited to $30 \%$ of total DRPTN studies and in $70 \%$ we were not able to identify explicit argument to support the validation phase of DRPTN models. Alternatively, we observed that eight studies (20\%) provide hypothetical numerical examples to validate their models. However, an illustrative example might not provide sufficient evidence to conclude the validity, quality or applicability of the entire model because it is tested within a simplified network and based on several uncertain assumptions, stochastic input data, subjective values and preferences, scenario-based damage states, and static traffic distribution. Scenario-based numerical case studies (with real-world data) can provide some level of confidence in the mathematical configuration of the algorithm and are beneficial in the sense of invalidating the model that initiates the advancement of the model construction in each invalidation process (Popper 1987). Using terms such as 'effective', 'validated, and 'accredit' for evaluating developed models based on internal properties of a model can yield misleading expectations from the model for future research and users of a publication in the sensitive critical context of DRPTN (Konikow and Bredehoeft 1992).

Furthermore, 11 studies (27.5\%) conduct a sensitivity analysis to evaluate the performance of models. Sensitivity analysis is indeed necessary to evaluate the input-dependency of the model as an observation over the internal behavior of the objective function to a supervised change in variables or weights. However, this observation, might offers very little in terms of insight or foresight about the validity of models to indicate its reliability to be used for a real-world instance of the indented problem (Oberkampf et al. 2003; Babu`ska and Oden 2004; Scandizzo 2016). Because sensitivity analysis neither evaluates the validity of the information fed into a model nor assesses the validity of the defined conceptual relationship among the models' parameters. Nevertheless, sensitivity analysis can sophisticatedly determine the degree of robustness of the model, logical relationships, or verify the algorithm. Therefore, although performing sensitivity observation is essential for DRPTN models, it should not be used interchangeably as a mechanism for the model validation in the DRPTN context.

A pressing need for the DRPTN field is to develop frameworks and approaches within the modeling process that provide a level of confidence in DRPTN models and pinpoint clear benchmarks within the validation process. One of these measures is to distinguish between subjective and objective parts of the models and control the subjectivity in the steps of the model construction. Another suggestion is to develop a set of critical conditions, tests, and boundaries in an attempt to refute DRPTN models or to quantify the uncertainty associated with the result of the model. Moreover, producing accredit synthetic observed data can also facilitate the "comparison of a known data to the known solution" 
that allows obtaining a satisfactory degree of credibility of models. This approach provides data from a simulated real world that can be compared to models' predictions. It, therefore, worth initiating efforts toward simulating a comprehensive visualized multi-dimensional, multi-disciplinary, agent-based post-disaster environment that can capture the complexity of the system and allow users to directly evaluate the consequences of different optimized recovery strategies.

\section{Summary and conclusions}

As a summary, first, we observed that DRPTN studies provide diverse sets of attributes in different categories, however, in a significant amount of cases, no conceptual underpinning has been presented to justify or support the selection process of the model's attributes. Therefore, it is essential that future studies propose systematic bias-reduced methods and methodologies toward establishing the attribute set of DRPTN problems and conduct a solid problem structuring. Second, despite successful divers formulations of the recovery problem, we could not identify an integration of traffic management and the disaster recovery problem and including traffic management options as a variable set of the decision model next to recovery projects. Hence, we proposed to integrate variables of route reopening and traffic control measures to reach the optimized performance of the transportation network. Third, we concluded that DRPTN problems require a computational, conceptual, and context-dependent justification for the selection and application sense-making of the solving methods. Therefore, we suggest devoting more effort to the identification of local characteristics of the problem with respect to complexity and convexity as a technical justification of utilizing deterministic and nondeterministic methods. Obviously, aiming at global solutions in the DRPTN context, if there is any, would promote the reliability of the model. In the last section, the study identified the major focus on verification of the optimization algorithms. Developing a systematic approach to provide a degree of confidence in the quality of non-observable models' solution remains a compelling direction for future works. Accordingly, the field of disaster recovery of infrastructures is calling for high-resolution simulations of the urban system in a microscopic level that facilitates modeling the individual decisionmaking process, within its sub-models and assimilates the user-user and user-system interactions in the post-disaster scenarios.

Finally, we pose this question that whether we need more novel, fast, and feasible optimization algorithms regardless of their conceptual and methodological strength in supporting the rationale of models' elements. Rather, research directions that can introduce science-developed-but-practice-oriented models which provide error-minimized practical results for operational levels. Perhaps what the field of DRPTN needs is not a new optimization approach or solving technique but conceptual descriptive models and systematic frameworks that stand as a solid foundation for models' construction and structuring in problem definition, solving and validation phases to avoid a method-richbut-methodology-poor phenomenon in the optimization-based DRPTN context. 


\section{Supplementary document}

The detailed report of the content analysis results within 40 publications based on attributes, types of problems, formulation approaches, and solving algorithms is available on TU Berlin server, Depositonce, accessible on https://dx.doi.org/10.14279/depos itonce-9077 as the supplementary document of this paper.

Acknowledgments Open Access funding provided by Projekt DEAL.

Open Access This article is licensed under a Creative Commons Attribution 4.0 International License, which permits use, sharing, adaptation, distribution and reproduction in any medium or format, as long as you give appropriate credit to the original author(s) and the source, provide a link to the Creative Commons licence, and indicate if changes were made. The images or other third party material in this article are included in the article's Creative Commons licence, unless indicated otherwise in a credit line to the material. If material is not included in the article's Creative Commons licence and your intended use is not permitted by statutory regulation or exceeds the permitted use, you will need to obtain permission directly from the copyright holder. To view a copy of this licence, visit http://creativecommons.org/licenses/by/4.0/.

\section{References}

Abdelgawad H, Abdulha IB (2009) Emergency evacuation planning as a network design problem: a critical review. Transp Lett. https://doi.org/10.3328/TL.2009.01.01.41-58

Babu`ska I, Oden JT (2004) Verification and Validation in computational engineering and science: basic concepts. Comput Methods Appl Mech Eng 193:4057-4066

Babuška I, Nobile F, Tempone R (2007) Reliability of computational science. Numer Methods Partial Differ Equ. https://doi.org/10.1002/num.20263

Belton V, Stewart T (2010) Problem structuring and multiple criteria decision analysis, In: Ehrgott M., Figueira J., Greco S. (eds) Trends in Multiple Criteria Decision Analysis. International Series in Operations Research \& Management Science, vol 142. Springer, Boston, MA.

Belton V, Stewart T (2012) Multiple criteria decision analysis: an integrated approach. Springer, US

Bertsekas DP (2015) Convex Optimization Algorithms. Athena Scientific, Belmont

Beven KJ, Aspinall WP, Bates PD et al (2015) Epistemic uncertainties and natural hazard risk assessmentpart 1: a review of the issues. Nat Hazards Earth Syst Sci Discuss. https://doi.org/10.5194/nhess d-3-7333-2015

Blum C, Roli A (2003) Metanon-deterministic methods s in combinatorial optimization: Overview and conceptual comparison. ACM Comput Surv 35:268-308

Boyd S, Vandenberghe L (2004) Convex optimization. Cambridge University Press, Cambridge

Braess D (1968) Über ein Paradoxon aus der Verkehrsplanung. Unternehmensforschung 12:258-268

Buchanan JT, Henig EJ, Henig MI (1998) Objectivity and subjectivity in the decision making process. Ann Oper Res 80:333-345

Cavalieri F, Franchin P, Gehl P, Khazai B (2012) Quantitative assessment of social losses based on physical damage and interaction with infrastructural systems. Earthq Eng Struct Dyn. https://doi.org/10.1002/ eqe. 2220

Celik S, Corbacioglu S (2010) Role of information in collective action in dynamic disaster environments. Disasters. https://doi.org/10.1111/j.1467-7717.2009.01118.x

Chang ES, Nojima N (2001) Measuring post-disaster transportation system performance: the 1995 kobe earthquake in comparative perspective. Transp Res Part A 35:475-494

Chmutina K, von Meding J (2019) A dilemma of language: natural disasters in academic literature. Int J Disaster Risk Sci. https://doi.org/10.1007/s13753-019-00232-2

Cova TJ, Conger S (2004) Transportation hazards. In: Kutz M (ed) Handbook of transportation engineering. McGraw Hill, New York, pp 297-304

Day J, Iris AJ, Silva L (2009) Information flow impediments in disaster relief supply chains. J AIS 10:1

Dehghani MS, Flintsch GW, McNeil S (2013) Roadway network as a degrading system: vulnerability and system level performance. Transp Lett. https://doi.org/10.1179/1942786713Z.0000000006 
Dobler C (2011) Exceptional events in a transport simulation. In R. Leidl and A. K. Hartmann (eds.) Modern Computational Science 11: Simulation of Extreme Events. Lecture Notes from the 3rd International Summer School Oldenburg, August 15-26, 2011, 311-325, BIS-Verlag, Oldenburg.

Duque-Maya P, Sörensen K (2011) A GRASP metaheuristic methods to improve accessibility after a disaster. OR Spectr. https://doi.org/10.1007/s00291-011-0247-2

Ehrgott M (2005) Multicriteria optimization. Springer, Berlin

El-anwar O, Ye J, Orabi W (2016a) Efficient optimization of post-disaster reconstruction of transportation networks. J Comput Civil Eng. https://doi.org/10.1061/(ASCE)CP.1943-5487.0000503

El-anwar O, Ye J, Orabi W (2016b) Innovative linear formulation for transportation reconstruction planning. J Comput Civil Eng. https://doi.org/10.1061/(ASCE)CP.1943-5487.0000504

Faturechi R, Miller-Hooks E (2015) Measuring the performance of transportation infrastructure systems in disasters: a comprehensive review. J Infrastruct Syst 21(1):4014025

Fekete A (2019) Social Vulnerability (Re-)assessment in context to natural hazards: review of the usefulness of the spatial indicator approach and investigations of validation demands. Int J Disaster Risk Sci. https://doi.org/10.1007/s13753-019-0213-1

Feng C, Wang T (2003) Highway emergency rehabilitation scheduling in post-earthquake 72 hours. J East Asia Soc Transp Stud 5(3281):20-28

Fernandes C, Pontes AJ, Viana JC, Gaspar-Cunha A (2018) Modeling and optimization of the injectionmolding process: a review. Adv Polym Technol. https://doi.org/10.1002/adv.2168

Festa P (2014) A brief introduction to exact, approximation, and heuristic algorithms for solving hard combinatorial optimization problems. In 2014 16th International Conference on Transparent Optical Networks (ICTON). pp 1-20. https://doi.org/10.1109/ICTON.2014.6876285

French M (2018) Fundamentals of Optimization. International Publishing, Springer

Galindo G, Batta R (2013) Review of recent developments in OR/MS research in disaster operations management. Eur J Op Res. https://doi.org/10.1016/j.ejor.2013.01.039

Grötschel M, Holland O (1991) Solution of large-scale symmetric travelling salesman problems. Math Program. https://doi.org/10.1007/BF01586932

Ha Mh, Yang Z (2018) Modelling interdependency among attributes in MCDM: Its Application in Port Performance Measurement In: P.T.-W. Lee, Z. Yang (eds.), Multi-Criteria Decision Making in Maritime Studies and Logistics, International Series in Operations Research \& Management Science 260,

Hackl J, Adey BT, Lethanh N (2018) Determination of near-optimal restoration programs for transportation networks following natural hazard events using simulated annealing. Computer-Aided Civil Infrastruct Eng. https://doi.org/10.1111/mice.12346

Horner MW, Widener MJ (2011) The effects of transportation network failure on people's accessibility to hurricane disaster relief goods: a modeling approach and application to a Florida case study. Nat Hazards. https://doi.org/10.1007/s11069-011-9855-z

Horst R, Tuy H (1996) Global Optimization. Springer, Berlin. https://doi.org/10.1007/978-3-662-03199-5

Iida Y, Kurauchi F, Shimada H (2000) Traffic management system against major earthquakes. IATSS Res 24(2):1-6

Ishibuchi H, Tsukamoto N, Yusuke N (2008) Evolutionary many-objective optimization: A short review. 2008 IEEE congress on evolutionary computation (IEEE World Congress on Computational Intelligence). Hong Kong. https://doi.org/10.1109/CEC.2008.4631121

Johannes J (2011) Vector optimization, theory, applications, and extensions. Springer-Verlag, Berlin Heidelberg

Kadri F, Birregah B, Châtelet E (2014) The impact of natural disasters on critical infrastructures: a domino effect-based study. J Homel Secur Emerg Manag. https://doi.org/10.1515/jhsem-2012-0077

Karlaftis GM, Kepaptsoglou KL, Lambropoulos S, Karlaftis MG, Kepaptsoglou KL, Lambropoulos S (2007) Fund Allocation for Transportation Network Recovery Following Natural Disasters. J Urban Plan Dev 133:82-89. https://doi.org/10.1061/(ASCE)0733-9488(2007)133:1(82)

Kaviani A, Thompson RG, Rajabifard A, Sarvi M (2018) A model for multi-class road network recovery scheduling of regional road networks. Transportation. https://doi.org/10.1007/s11116-017-9852-5

Keeney R (1992) Value focused thinking: a path to creative decision making. Harvard University Press, Cambridge

Keeney R, Gregory R (2005) Selecting attributes to measure the achievement of objectives. Op Res 53:1-11

Koks E, Rozenberg J, Zorn C et al (2019) A global multi-hazard risk analysis of road and railway infrastructure assets. Nat Sustain 10(1):1-11

Konikow LF, Bredehoeft JD (1992) Groundwater models cannot be validated? Adv Water Resourc 15:75-83

Konstantinidou MA, Kepaptsoglou KL, Karlaftis MG (2014) Transportation network post-disaster planning and management: a review part II: Decision-making and planning of post-disaster operations. Int J Transp 2(3):17-32 
Lange K (2013) Optimization, 2nd edn. Springer, New York. https://doi.org/10.1007/978-1-4614-5838-8

Lertworawanich P (2012) Highway network restoration after the great flood in Thailand. Nat Hazards. https ://doi.org/10.1007/s11069-012-0278-2

Leskens JG, Brugnach M, Hoekstra A, Schuurmans W (2014) Why are decisions in flood disaster management so poorly supported by information from flood models. Environ Modell Software. https://doi. org/10.1016/j.envsoft.2013.11.003

Liao T, Hu T, Ko Y (2018) A resilience optimization model for transportation networks under disasters. Nat Hazards. https://doi.org/10.1007/s11069-018-3310-3

Liefooghe A (2011) Metanon-deterministic methods s for multiobjective optimisation: Cooperative approaches, uncertainty handling and application in logistics 4OR: A Quarterly Journal of Operations Research, Springer Verlag, 9 (2), pp.219-222.

Limbourg P (2005) Multi-objective Optimization of Problems with Epistemic Uncertainty BT - Evolutionary Multi-Criterion Optimization. In C. A. Coello Coello, A. Hernández Aguirre, \& E. Zitzler (Eds.) (pp. 413-427). Springer, Berlin.

Lubashevskiy V, Suzuki T, Kanno T, Furuta K (2017) Recovery of urban socio-technical systems after disaster: quasi-optimality of reactive decision-making based planning. EURO J Decis Process. https:// doi.org/10.1007/s40070-017-0066-z

Marler R, Arora J (2004) Survey of multi-objective optimization methods for engineering. Struct Multidisc Optim 26:369

Mart'1 R, Reinelt G (2011) The linear ordering problem, exact and heuristic methods in combinatorial. Optimization. https://doi.org/10.1007/978-3-642-16729-43

Mitroff II, Featheringham TR (1974) On systemic problem solving and the error of the third kind. Behav Sci 19:383-393

Morris WT (1967) On the art of modeling. Manage Sci 13(12):B707-717

Naga P, Fan YY (2007) Quick estimation of network performance measures using associative memory techniques. Transp Res Rec 2039:75-82

National Research Council (NRC) (1999) Reducing disaster losses through better information. National Academy Press, Washington

Nigg JM (1995) Disaster recovery as a social process. Wellington after the quake: The challenge of rebuilding. The Earthquake Commission, Wellington, pp 81-92

Nocedal J, Wright S (1999) Numerical optimization. Springer, New York

Oberkampf W, Roy C (2010) Verification and validation in scientific computing. Cambridge University Press, Cambridge

Oberkampf WL, Trucano TG, Hirsch Ch (2003) Verification, validation, and predictive capabilities in computational engineering, and physics, Tech. Rep. Sand. No. 2003-3769, SANDIA.Nat.Lab.

Orabi W, El-rayes K, Senouci AB, Al-derham H (2009) Optimizing postdisaster reconstruction planning for damaged transportation networks. J Constr Eng Manag. https://doi.org/10.1061/(ASCE)CO.19437862.0000070

Orabi W, Senouci AB, El-Rayes K, Al-Derham H (2010) Optimizing resource utilization during the recovery of civil infrastructure systems. J Manag Eng. https://doi.org/10.1061/(ASCE)ME.19435479.0000024

Phillips-Wren G, Power DJ, Mora M (2019) Cognitive bias, decision styles, and risk attitudes in decision making and DSS. J Decis Syst. https://doi.org/10.1080/12460125.2019.1646509

Phillips LD (1984) A theory of requisite decision models, Acta Psychol, 56(1-3), 29-48, ISSN 0001-6918.

Popper K (1987) Science: Conjectures and refutations. In: Kourany JA (ed) Scientific knowledge: Basic issues in the philosophy of science. Wadsworth, Belmont, pp 139-157

Rardin RL, Uzsoy R (2001) Experimental evaluation of heuristic optimization algorithms: a tutorial. J Nondeterministic Methods s. https://doi.org/10.1023/A:1011319115230

Renne J, Wolshon B, Murray-Tuite P, Pande A (2020) Emergence of resilience as a framework for state Departments of Transportation (DOTs) in the United States. Transp Res Part D Transp Environ 82:102178

Rockafellar, R.T., 1997. Fundamentals of optimization. University of Washington. Available: https://www. math.washington.edu/ burke/crs/515/

Rothlauf F (2011) Design of modern heuristics. Nat Comput Ser. https://doi.org/10.1007/978-3-540-72962 $-49$

Roy CJ, Oberkampf WL (2011) A comprehensive framework for verification, validation, and uncertainty quantification in scientific computing. Comput Methods Appl Mech Eng 200(25):2131-2144

Sargent RG (1996) Verifying and validating simulation models, In Proceedings 1996 Winter Simulation Conf. IEEE Computer Society Press, pp 55-64. 
Sargent RG (2011) Verification and validation of simulation models, In Proceedings of the 2011 Winter Simulation Conference (WSC).

Sato T, Ichii K (1995) Optimization of post-earthquake restoration of lifeline networks using genetic algorithms, In Proceedings of the Sixth U.S.-Japan workshop on earthquake disaster prevention for lifeline systems. Public Works Research Institute, Osaka

Scandizzo S (2016) The validation of risk models. A handbook for practitioners. Palgrave Macmillan, London

Schneider J, Kirkpatrick S (2006) Stochastic Optimization. Springer, Berlin. https://doi.org/10.1007/978-3540-34560-2

Shiraki W, Takahashi K, Inomo H, Isouchi C (2017) A proposed restoration strategy for road networks after an earthquake disaster using resilience engineering. J Disaster Res. https://doi.org/10.20965/jdr.2017. p0722

Taha HA (2007) Operations research. An introduction, 8th edn. Macmillan Publishing Company, New York

Talbi E, Basseur M, Nebro AJ, Alba E (2012) Multi-objective optimization using metanon-deterministic methods s: non-standard algorithms. Int Trans Op Res. https://doi.org/10.111 1/j.1475-3995.2011.00808.x

Tiesmeier DK (2016) MCDM problem-structuring framework and a real estate decision support model. University of Manchester, England

Tovey CA (2002) Tutorial on computational complexity. Interfaces. https://doi.org/10.1287/inte.32.3.30.39

Udy J, Hansen B, Maddux S et al (2017) Review of field development optimization of water flooding, eor, and well placement focusing on history matching and optimization algorithms. Process 5:34

Unal M, Warn GP (2015) A many-objective framework to design the restoration of damaged bridges on a distributed transportation network. Struct Congr. https://doi.org/10.1061/9780784479117.128

Vianna DS, Vianna M (2013) Local search-based non-deterministic methods s for the multiobjective multidimensional knapsack problem. Production. https://doi.org/10.1590/S0103-65132012005000081

Wang J, Qiao C, Yu H (2011) On progressive network recovery after a major disruption. Proc IEEE INFOCOM Shanghai 2011:1925-1933. https://doi.org/10.1109/INFCOM.2011.5934996

Wardrop JG (1952) Some theoretical aspects of road traffic research, In Proceedings of the Institute of Civil Engineers. Part II, pp. 325-378

Williams HP (2013) Model building mathematical programming, 5th edn. John Wiley \& Sons Ltd, Hoboken

Winter B, Schneeberger K et al (2018) Sources of uncertainty in a probabilistic flood risk model. Nat Hazards 91:431-446

Wu X, Li C, He Y, Jia W (2018) Operation optimization of natural gas transmission pipelines based on stochastic optimization algorithms: a review. Math Prob Eng. https://doi.org/10.1155/2018/1267045

Xing H (2017) The decision method of emergency supplies collection with fuzzy demand constraint under background of sudden disaster. Nat Hazards. https://doi.org/10.1007/s11069-016-2608-2

Xu X, Huang Y, Chen K (2019) Method for large group emergency decision making with complex preferences based on emergency similarity and interval consistency. Nat Hazards. https://doi.org/10.1007/ s11069-019-03624-1

Yin C (2020) Hazard assessment and regionalization of highway flood disasters in China. Nat Hazards 100:535-550

Yuan JL, Yan YD, Huang D, Du C (2014) Restoration strategies of urban road network after earthquake based on corrected component probabilistic importance. Appl Mech Mater. https://doi.org/10.4028/ www.scientific.net/AMM.694.95

Zamanifar M, Seyedhoseyni SM (2017) Recovery planning model for roadways network after natural hazards. Nat Hazards. https://doi.org/10.1007/s11069-017-2788-4

Zhang RH (1992) Lifeline interaction and post-earthquake urban system reconstruction, Proceedings of 10th WCEE, pp. 5475-5480. Eshghi S ed. Rotterdam: A Balkema Publishers.

Zhang TZ, Lu YM (2011) Study on simulation and optimization of the road rush-repair model after disaster. Appl Mech Mater 50-51:298-303

Zhu X, Zhang G, Sun B (2019) A comprehensive literature review of the demand forecasting methods of emergency resources from the perspective of artificial intelligence. Nat Hazards 97:65-82. https://doi. org/10.1007/s11069-019-03626-z

Zopounidis C, Doumpos M (2002) Multi-criteria decision aid in financial decision making: methodologies and literature review. J Multi-Criteria Decis Anal 11:167-186

Publisher's Note Springer Nature remains neutral with regard to jurisdictional claims in published maps and institutional affiliations. 